\section{Anti-EGFR Therapy in Right-Sided Metastatic Colorectal Cancer: Right or Wrong?}

\author{
Benjamin A. Weinberg, MD
}

Colorectal cancer (CRC) is the second leading cause of cancer-related death in the United States after lung cancer, and American Cancer Society sources estimate that more than 50,000 Americans will die of CRC this year alone. ${ }^{1}$ In addition to chemotherapy, biologic agents are an important component of the arsenal against metastatic CRC (mCRC). Use of cetuximab and panitumumab, which are monoclonal antibodies targeting the epidermal growth factor receptor (EGFR), has evolved substantially over the past 2 decades. ${ }^{2}$ Immunohistochemical evidence of EGFR-positivity in tumors was initially thought to be an appropriate therapeutic biomarker, but this EGFR expression was not predictive of response to cetuximab, ${ }^{2}$ and the response rate for patients with EGFR-positive chemorefractory mCRC treated with cetuximab was only $8 \% .^{3}$ Further work demonstrated that cetuximab treatment outcomes were worse for patients with tumor mutations in KRAS exon 2 than for their wild-type counterparts. ${ }^{4}$ However, KRAS exon 2 mutations are only part of the picture; thus, patients with mutations in KRAS exons 3-4 and NRAS exons 2-4 also do not benefit from anti-EGFR therapy., 5 Patients with BRAF V600E mutations likewise do not benefit from EGFR inhibitors unless combined with irinotecan and BRAF-targeted therapies.

More recently, tumor sidedness has emerged as another predictive biomarker for response to cetuximab and panitumumab in the RAS and BRAF wild-type population. Patients with mCRC and right-sided primary tumors (cecum, ascending and proximal transverse colon) have inferior overall survival compared with patients with mCRC and left-sided primaries (distal transverse, descending, and sigmoid colon and rectum). ${ }^{7}$ Moreover, patients with right-sided primaries do not benefit from cetuximab in the firstline setting, whereas patients with left-sided primaries clearly benefit. ${ }^{8}$

In the CALGB/SWOG 80405 study of first-line chemotherapy plus cetuximab versus chemotherapy plus bevacizumab in patients with KRAS exon 2 wild-type mCRC, the longest overall survival was seen in the study arm that included patients with leftsided tumors treated with cetuximab (36.0 months), followed by the left-sided tumors treated with bevacizumab arm (31.4 months), followed by the right-sided tumors treated with bevacizumab arm (24.2 months), and finally the right-sided tumors treated with cetuximab arm (16.7 months). ${ }^{8}$ Although cetuximab led to worse outcomes in patients with right-sided tumors regardless of KRAS status, it can be suggested that patients with RAS and BRAF wild-type left-sided primary tumors should receive anti-EGFR therapy in the first line. Perhaps the treatment sequence is important in this latter case, and EGFR therapy upfront improves response to future lines of therapy.

These findings raise some inevitable questions: can we ever use anti-EGFR drugs in the treatment of patients with right-sided mCRC, particularly in those with RAS and BRAF wild-type tumors, as a later line of therapy, and what other biomarkers can we use to inform this decision? In this issue of JNCCN, Kratz et al attempt to answer this question by looking at tumor bulk using $3.5 \mathrm{~cm}$ as a cutoff. In a small retrospective analysis in the late-line setting, these authors show that anti-EGFR therapies work best in nonbulky (small) tumors, especially when the tumors are on the left side. Most importantly, nonbulky right-sided mCRCs respond to anti-EGFR therapy, but only with the addition of chemotherapy.

Small sample numbers make drawing definitive conclusions difficult, but these results suggest that anti-EGFR monotherapy should be avoided in patients with nonbulky rightsided mCRC and that anti-EGFR therapy, whether alone or in combination, should be avoided altogether in patients with bulky right-sided mCRC. The authors reasonably

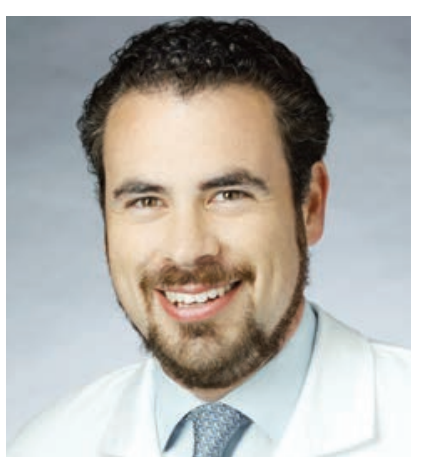

Benjamin A. Weinberg, MD

Benjamin A. Weinberg, MD, is an Assistant Professor of Medicine in the Ruesch Center for the Cure of Gastrointestinal Cancers, Division of Hematology and Oncology, Lombardi Comprehensive Cancer Center, Georgetown University. doi: 10.6004 ljnccn.2018.7107

The ideas and viewpoints expressed in this commentary are those of the author and do not necessarily represent any policy, position, or program of NCCN. 
Weinberg

propose that as tumor bulk increases, the anti-EGFR monoclonal antibodies are less likely to reach their target on the tumor cell surface. Tumor bulk should theoretically not present a similar issue for bevacizumab, which binds to soluble vascular endothelial growth factor A (VEGF-A) in the extracellular space, not on the tumor cell surface.

Putting bulk aside, what is the biological explanation for tumor sidedness affecting response to anti-EGFR therapies in patients with RAS and BRAF wild-type mCRCs? The right- and left-colon arise from different embryologic structures (midgut and hindgut, respectively) and have different blood supplies (superior and inferior mesenteric arteries, respectively). Tumor mutational profiles vary throughout the colon, with higher rates of BRAF - and PIK3CA-mutated and microsatellite unstable tumors on the right side and higher rates of TP53 - and APC-mutated and HER2-amplified tumors on the left side and in the rectum. Importantly, mutational profiles continuously change throughout the colon, and there is not a dichotomous split between right and left. Indeed, recent work suggests that tumor profiles vary across specific regions in the right colon, left colon, and rectum, further complicating matters. ${ }^{9}$

What causes such genomic diversity in CRC? Part of the answer lies in the colonic microbiome, which varies among individuals, age groups, and locations within the colon and rectum. Certain culprit bacteria such as Fusobacterium nucleatum, Bacteriodes fragilis, and Streptococcus gallolyticus have known roles in CRC carcinogenesis. ${ }^{10}$ More research is necessary to elucidate whether certain carcinogenic bacteria give rise to CRC with more or less sensitivity to anti-EGFR drugs. Bacteria may be the missing link accounting for the different tumor biology of the right colon that leads to resistance to anti-EGFR therapy.

For now, the right colon is probably the wrong place to consider using single-agent cetuximab or panitumumab. The impulse to use these agents when all other available therapies have been exhausted should be countered by the concern that EGFR blockade in this setting may actually result in worse patient outcomes. Right-sided mCRC tumors that are RAS and BRAF wild-type and nonbulky may respond to combination chemotherapy with anti-EGFR antibodies, but not to anti-EGFR therapy alone. Future efforts need to address the mechanisms of resistance to anti-EGFR therapy in right-sided RAS, BRAF, and PIK3CA wild-type mCRC.

\section{Acknowledgments}

The author thanks Marion Hartley, PhD, Science Writer for Clinical Research at the Ruesch Center for the Cure of Gastrointestinal Cancers, Lombardi Comprehensive Cancer Center, Georgetown University, for her edits and suggestions during the composition of this editorial.

\section{References}

1. American Cancer Society. Cancer Facts \& Figures. Atlanta: American Cancer Society; 2018

2. Cunningham D, Humblet $Y$, Siena S, et al. Cetuximab monotherapy and cetuximab plus irinotecan in irinotecanrefractory metastatic colorectal cancer. N Engl J Med 2004;351:337-345.

3. Jonker DJ, O'Callaghan CJ, Karapetis CS, et al. Cetuximab for the treatment of colorectal cancer. N Engl J Med 2007;357:2040-2048.

4. Van Cutsem E, Kohne $\mathrm{CH}$, Hitre E, et al. Cetuximab and chemotherapy as initial treatment for metastatic colorectal cancer. N Engl J Med 2009;360:1408-1417.

5. Douillard JY, Oliner KS, Siena S, et al. Panitumumab-FOLFOX4 treatment and RAS mutations in colorectal cancer. N Engl J Med 2013;369:1023-1034.

6. Di Nicolantonio F, Martini M, Molinari F, et al. Wild-type BRAF is required for response to panitumumab or cetuximab in metastatic colorectal cancer. J Clin Oncol 2008;26:5705-5712.

7. Loupakis F, Yang D, Yau L, et al. Primary tumor location as a prognostic factor in metastatic colorectal cancer. J Natl Cancer Inst 2015;107:dju427.

8. Venook AP, Ou FS, Lenz HJ, et al. Primary tumor location as an independent prognostic marker from molecular features for overall survival in patients with metastatic colorectal cancer: analysis of CALGB/SWOG 80405 (Alliance) [abstract]. J Clin Oncol 2017;35(Suppl):Abstract 3503.

9. Loree JM, Pereira AA, Lam M, et al. Classifying colorectal cancer by tumor location rather than sidedness highlights a continuum in mutation profiles and consensus molecular subtypes. Clin Cancer Res 2018;24:10621072 .

10. Sears CL, Garrett WS. Microbes, microbiota, and colon cancer. Cell Host Microbe 2014;15:317-328. 